\title{
INTERVENÇÃO PROFISSIONAL NOS ESPORTES DE AVENTURA: UMA PERSPECTIVA CONCEITUAL À FORMAÇÃO E À INVESTIGAÇÃO
}

\author{
PROFESSIONAL INTERVENTION IN ADVENTURE SPORTS: A CONCEPTUAL \\ PERSPECTIVE FOR EDUCATION AND RESEARCH
}

\author{
INTERVENCIÓN PROFESIONAL EN LOS DEPORTES DE AVENTURA: UNA \\ PERSPECTIVA CONCEPTUAL A LA FORMACIÓN E INVESTIGACIÓN
}

Vinicius Zeilmann Brasil*, Valmor Ramos ${ }^{\star *}$, Juarez Vieira do Nascimento*

\begin{abstract}
Palavras chave:
Treinador.

Educação Física

e Treinamento.

Esportes.

Natureza.

Resumo: 0 objetivo deste ensaio teórico foi apresentar e discutir um conjunto de conceitos que possibilitem uma compreensão abrangente da intervenção profissional nos esportes de aventura. A delimitação conceitual adotada para os esportes de aventura abrange as modalidades que já se configuram como possibilidade de intervenção pedagógica no âmbito esportivo. 0 modelo conceitual sobre o Treinador de Esportes de Aventura (TEA), proposto por Collins e Collins (2015), tem influência dos marcos teóricos utilizados para investigações sobre o treinador esportivo. Seus componentes estruturantes abrangem 0 foco da intervenção, a capacidade do TEA para se manter no ambiente natural, o processo de gestão do risco e tomada de decisão e, também, uma estrutura epistemológica pessoal que fundamenta sua intervenção. A análise desses componentes, em diferentes realidades de intervenção, compreende uma possibilidade de produção de conhecimento e de diálogo científico com implicações na qualificação profissional e reconhecimento social da atividade do TEA.
\end{abstract}

\section{Keywords:}

Coach.

Physical Education and Training.

Sports.

Nature.

\begin{abstract}
This study presents and discusses a set of concepts to provide a broad understanding about professional intervention in adventure sports. The conceptual delimitation adopted for adventure sports includes those sports that are possibilities for pedagogical intervention in sports. Collins and Collins's (2015) Adventure Sports Coach Model (ASC) was designed under the influence of theoretical frameworks used in sports coach research. The Model's components include the intervention focus, ASC's ability to remain in the natural environment, risk management and decision-making processes, and the personal epistemological structure at the basis of their intervention. The analysis of these conceptual components in different contexts can be useful for knowledge production and scientific interchange with implications on ASC's development and social recognition.
\end{abstract}

Palabras clave: Entrenador. Educación Física y Entrenamiento. Deportes. Naturaleza.
Resumen: El objetivo de este ensayo teórico fue presentar y discutir un conjunto de conceptos que posibiliten una comprensión amplia de la intervención profesional en los deportes de aventura. La delimitación conceptual adoptada para los deportes de aventura abarca modalidades que ya se configuran como posibilidad de intervención pedagógica en el ámbito deportivo. El modelo conceptual sobre el Entrenador de Deportes de Aventura (EDA), propuesto por Collins y Collins (2015), está influenciado por los marcos teóricos utilizados para investigaciones sobre el entrenador deportivo. Sus componentes estructurales abarcan el foco de la intervención, la capacidad del EDA para mantenerse en el ambiente natural, el proceso de gestión del riesgo y de toma de decisión, y una estructura epistemológica personal que fundamenta su intervención. El análisis de estos componentes, en diferentes realidades de intervención, comprende una posibilidad de producción de conocimiento y de diálogo científico con implicaciones en la calificación profesional y reconocimiento social de la actividad del EDA.
*Universidade Federal de Santa de Santa Catarina. Florianópolis, SC, Brasil.

E-mail: vzbrasil@hotmail.com; juarez.nascimento@ufsc.br

**Universidade do Estado de Santa Catarina. Florianópolis, SC, Brasil. E-mail: valmor.ramos@udesc.br

Recebido em: 13-06-2018 Aprovado em: 26-08-2018 Publicado em: 13-03-2019

DOl: https://doi.org/10.22456/1982-8918.83842

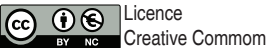




\section{INTRODUÇÃOO}

A intervenção profissional nos esportes de aventura tem ocorrido, com predomínio, no campo do lazer, turismo e, mais recentemente, em contextos de prática esportiva para a iniciação e rendimento esportivo. No contexto brasileiro, estudos com instrutores de surfe, rafting, parapente, condutores de trilhas, entre outras modalidades, evidenciam a valorização do domínio das habilidades esportivas (PAIXÃO et al., 2011; RAMOS; BRASIL; GODA, 2013; SCHWARTZ; CARNICELLI FILHO, 2006), do conhecimento detalhado acerca do ambiente natural (PAIXÃO; SILVA, 2017) e da capacidade para gerir o risco inerente a essas modalidades (BRASIL et al., 2016; PAIXÃO; GEIDEL, 2018). Isso se traduz na capacidade de o profissional criar situações de prática esportiva prazerosas que garantam integridade física e emocional dos praticantes, assegurando a preservação do meio natural em que atua (CARVALHINHO et al., 2010; PAIXÃO, 2012).

A informalidade das vias de inserção social dos esportes de aventura e 0 caráter multidisciplinar da orientação e do ensino dessas modalidades dificultam a definição da área profissional à qual tais práticas esportivas estariam vinculadas $\mathrm{e}$, consequentemente, das oportunidades formativas e de certificação profissional para intervir nesses esportes (BANDEIRA; RIBEIRO, 2015; VAZ et al., 2017). Esse quadro tem sido um desafio para a produção e 0 debate científico, bem como para definição de um conjunto de conhecimentos e procedimentos, validados cientificamente, que oriente a atuação profissional nos esportes de aventura, com implicações na formação de futuros profissionais e na legitimação dessa atividade.

A perspectiva que se apresenta neste ensaio teórico situa a prática dos esportes de aventura como parte de um fenômeno sociocultural esportivo amplo, abrangendo um campo de intervenção emergente aos profissionais da área de Educação Física e Esporte. Acreditase que a demanda por determinado nível de desempenho físico e esportivo e a presença do componente pedagógico na orientação e no ensino dos esportes de aventura permitem a análise da intervenção profissional nessas modalidades a partir de orientações teóricas e metodológicas que têm delimitado objetos de estudo e a base de conhecimentos para a intervenção profissional no âmbito do ensino e do treino esportivo em geral.

Assim sendo, a intervenção profissional nos esportes de aventura é analisada a partir da proposta conceitual de Collins e Collins $(2012,2015)$ sobre o Treinador de Esportes de Aventura (TEA) (Adventure sports coach model, como um subgrupo emergente no contexto de intervenção do treinador esportivo. Sob essa perspectiva, estudos recentes com treinadores de paddle sports, surfe, kayaking, canoagem, montanhismo, escalada e mountain biking evidenciam que para explorar os benefícios pedagógicos dos ambientes desafiadores (natureza) os treinadores necessitam um desempenho técnico na modalidade, competências de coaching, uma estrutura epistemológica sofisticada (CHRISTIAN; BERRY; KEARNEY, 2017; COLLINS; COLLINS; GRECIC, 2015) e uma capacidade para tomada de decisão precisa (COLLINS; COLLINS, 2013; 2015a; 2017).

Essa proposta, no âmbito internacional, é uma possibilidade de análise e discussão, acenando a necessidade de ampliação das evidências científicas com implicações na sistematização de um conjunto de conhecimentos a respeito da intervenção do TEA. Enquanto objeto de investigação, no contexto brasileiro, a intervenção nos esportes de aventura não demonstra uma perspectiva conceitual clara para se analisar o componente pedagógico presente nessa atividade profissional. 0 que há, ainda, é uma atuação profissional fundamentada nas 
experiências de prática esportiva (PAIXÃO; GEIDEL, 2018; PAIXÃO; TUCHER, 2010) e 0 predomínio da qualificação técnica oferecida por agências de turismo e entidades esportivas (AURICCHIO, 2017; BANDEIRA; RIBEIRO, 2015).

Desta forma, pretende-se por meio deste ensaio teórico apresentar e discutir um conjunto de conceitos que possibilitem uma compreensão abrangente da intervenção profissional nos esportes de aventura, no sentido de fornecer uma perspectiva conceitual que possa estimular o debate, a produção científica e a formação profissional na área. Para tanto, serão apresentadas uma delimitação conceitual para os esportes de aventura e as possibilidades de intervenção profissional nessa área; a definição de Treinador de Esportes de Aventura (TEA) e os componentes de sua intervenção profissional a partir da proposta Conceptualizing the adventure sports coach model (COLLINS; COLLINS, 2012; 2015); e as implicações da perspectiva conceitual apresentada para o desenvolvimento profissional e para a investigação científica acerca do TEA.

\section{A INTERVENÇÃO PROFISSIONAL NOS ESPORTES DE AVENTURA}

A busca pela prática de esportes de aventura, sobretudo em ambientes naturais, integra uma dinâmica evolutiva social e esportiva, com implicações na relação do homem com a natureza, equivalente ao processo de gênese de identidades vinculadas a essas práticas (THORPE; WHEATON, 2011; WHEATON, 2004). A influência de diferentes técnicas e tradições esportivas caracteriza a inserção social dessas modalidades, abrangendo também a produção de símbolos de identificação coletiva, no fluxo de ideias, imagens e produtos compartilhados pelos praticantes (DIAS, MELO; ALVES JÚNIOR, 2007). Além disso, a convivência entre 0 esportista e a natureza institui a busca de novos valores e significados para a prática esportiva, marcada por sentimentos de autorrealização, autoexpressão e liberdade (MARINHO, 2008).

Essas manifestações esportivas se caracterizam por apresentar um caráter distinto das concepções tradicionais de prática esportiva, sendo designadas a partir de diversas terminologias, a saber: Atividades Físicas de Aventura na Natureza (BETRÁN; BETRÁN, 1995); Atividades Esportivas em Meio Natural (FUNOLLET, 1995); Esportes Radicais (UVINHA, 2001); Esportes na Natureza (DIAS, 2007); Atividades de Aventura (PIMENTEL, 2013); Práticas Corporais de Aventura na Natureza (INÁCIO, 2014); Extreme Sports (BRYMER, 2013); Lifestyle Sports (WHEATON, 2004); e Adventure Sports (BREIVIKI, 2010).

A denominação Esportes de Aventura (Adventure Sports) abrange duas orientações conceituais emergentes. Uma que enfatiza a ação motriz do praticante em atividades esportivas que apresentam um nível de desafio estabelecido pelo próprio esportista e que ocorrem em ambientes exigentes (naturais ou artificiais) e, também, o risco como um elemento constante e crítico da prática gerenciado pelo praticante, a independência e autonomia de prática e a ausência de regras arbitrárias. A outra orientação integrante diz respeito ao conceito de Lifestyle Sports, que compreende atividades individualistas, mas tendentes a criarem grupos e subculturas em torno da prática esportiva, promovendo valores e atitudes que priorizam a liberdade de expressão pessoal. Esse conceito foi proposto por Wheaton (2004), ao analisar práticas esportivas que congregam histórias e ideologias distintas da cultura esportiva tradicional (ao menos, em suas fases iniciais de desenvolvimento).

O termo Esportes de Aventura, portanto, faz referência a um amplo conjunto de modalidades esportivas, tendo, como exemplo, o skateboard, o windsurf, o surf, o parkour, 
o montanhismo, o snowboard, a escalada e o kitesurf, que trazem, em seu curso de desenvolvimento, elementos histórico-culturais presentes nos hábitos, crenças, atitudes e comportamentos dos praticantes. E embora cada uma dessas modalidades evidencie particularidades em termos de história, formas de inserção social e institucionalização, padrões de expressão e identidade, muitas delas compartilham um conjunto de atributos morais, sociais e afetivos (ethos) que as distinguem dos esportes tradicionais (BREIVIK, 2010).

As possibilidades de intervenção profissional nos esportes de aventura têm ocorrido com foco no lazer, no turismo e na iniciação esportiva de crianças, jovens e adultos. Particularmente, o surf, o skateboard, o snowboard, o kitesurf, o windsurf e a escalada esportiva são alguns exemplos de modalidades que já apresentam demanda para uma intervenção no âmbito da formação e do treinamento esportivo para competições de alto rendimento (ELLMER; RYNNE, 2018; TAYLOR; MCEWAN, 2015). No Brasil, a atuação profissional nestas práticas esportivas tem se caracterizado a partir de duas perspectivas distintas, o Turismo de Aventura e os Esportes de Aventura (MARINHO; UVINHA, 2009).

A primeira corresponde ao conceito difundido pelo Ministério do Turismo, fazendo referência às atividades turísticas que envolvem a prática de esportes de aventura em meio natural, as quais possuem fins comerciais e de caráter não competitivo, ofertadas por empresas ou agências de turismo. A intervenção profissional, neste caso, compreende o acompanhamento e orientação do turista nas atividades de aventura oportunizando novas experiências, sensações, iniciativas e a interação com o ambiente. Há, neste contexto, um conjunto de normas técnicas oficiais que estabelecem aos profissionais uma série de orientações referentes a qualidade, funcionalidade e segurança do serviço prestado (MARINHO; UVINHA, 2009).

A perspectiva dos esportes de aventura abrange as práticas esportivas, geralmente na natureza, nas quais o esportista participa como agente ativo, sendo conhecedor ou não da atividade, praticada de forma espontânea em situações lazer, recreação ou competição (MARINHO; UVINHA, 2009). A intervenção, neste caso, está vinculada às iniciativas de profissionais autônomos ou a centros de treinamento esportivo e escolas especializadas (BANDEIRA; RIBEIRO, 2015). Mais recentemente, a iniciação de jovens esportistas e 0 treinamento para competições, por meio de programas de prática esportiva sistematizada, emergem como uma nova possibilidade de intervenção profissional nos esportes de aventura. Nesse contexto o profissional tem como finalidade maximizar experiências esportivas, desenvolver habilidades e formar esportistas autônomos (COLLINS; COLLINS, 2015; OJALA; THORPE, 2015).

Considerando o itinerário conceitual delineado para o presente estudo, considera-se, portanto, a intervenção profissional nos esportes de aventura sob a perspectiva que se tem analisado e conceituado o processo de treino ou coaching esportivo, a partir das matrizes teóricas e das investigações que têm aporte nas ciências do esporte. 0 interesse recai na tentativa de articular uma demanda atual de intervenção pedagógica nos esportes de aventura e uma perspectiva conceitual que possibilite incursões nessa temática.

\section{OS COMPONENTES CONCEITUAIS DA INTERVENÇÃO PROFISSIONAL DO TREINADOR DE ESPORTES DE AVENTURA (TEA)}

Embora se reconheça a indefinição a respeito da denominação do profissional que intervém no âmbito dos esportes de aventura, sendo identificado, frequentemente, como 
instrutor, professor, guia, orientador ou monitor, o termo Treinador de Esportes de Aventura (TEA) ou Adventure Sports Coach, proposto por Collins e Collins (2012), corresponde a uma possibilidade de delimitação conceitual dessa atividade profissional emergente no contexto esportivo atual. Em essência, o TEA é o profissional que tem o papel de desenvolver praticantes capazes de participar, de maneira autônoma, em suas próprias experiências esportivas de aventura, sejam elas voltadas ao lazer, como estilo de vida ou para o alto rendimento (COLLINS; COLLINS, 2015).

A intervenção do TEA caracteriza-se pela complexidade e pela instabilidade do meio em que a prática esportiva ocorre (na natureza) e, também, da especificidade dos equipamentos utilizados e das habilidades esportivas exigidas. $O$ risco inerente a essas modalidades opera como um elemento pedagógico determinante na intervenção do TEA e na aprendizagem dos praticantes, de modo que o estabelecimento de níveis adequados de risco apresenta implicações claras na motivação, aquisição das habilidades e segurança dos aprendizes (COLLINS; COLLINS, 2012; 2015).

A iniciativa conceitual de Collins e Collins (2012) de considerar a intervenção do TEA como um subgrupo do coaching esportivo implica, necessariamente, reconhecer um conjunto de crenças, pensamentos e procedimentos típicos da intervenção pedagógica do treinador esportivo, já analisados e conceituados nos esportes tradicionais, acrescido dos elementos específicos da atuação nos esportes de aventura. Portanto, essa perspectiva conceitual é uma possibilidade para estabelecer uma linguagem consensual e uma série de pressupostos que preenchem uma lacuna de estudos e conceituação sobre a intervenção profissional nessa área.

Por definição, o coaching esportivo compreende um sistema complexo que envolve uma atividade social dinâmica, caracterizada pelo engajamento do treinador e do atleta em situações ligadas a uma determinada modalidade esportiva, com implicações na construção de identidade desses indivíduos (BOWES; JONES, 2006; CUSHION, 2007; LYLE, 2002). Nesta perspectiva, há o reconhecimento da natureza circunstancial e espontânea das ações do treinador esportivo e do esporte como espaço social no qual sua intervenção se situa (LYLE, 2018).

Diversas investigações (ABRAHAM; COLLINS; MARTINDALE, 2006; BARNSON, 2014; CÔTÉ et al., 1995) sob a perspectiva dos "models of coaching" propuseram modelos que representam a intervenção do treinador esportivo, especialmente aqueles elaborados a partir da análise da própria intervenção de treinadores experts (CUSHION; ARMOUR; JONES, 2006). As evidências empíricas obtidas nesses estudos contribuíram, de fato, para o entendimento sobre a natureza do coaching e da intervenção do treinador (coaching process), revelando parte da complexidade dessa atividade profissional, sobretudo, pela adoção de abordagens mais qualitativas.

Embora reconheçam as importantes contribuições das investigações sob essa perspectiva, Jones e Wallace (2005) destacam a necessidade de analisar a relação interdependente entre o treinador, o atleta e o contexto de treinamento, a fim de fornecer uma perspectiva mais realista e sofisticada do que realmente há na intervenção do treinador. A noção de interdependência consiste em um ponto importante para se compreender que nenhum elemento de sua intervenção pode determinar unilateralmente suas ações. Ou seja, as demandas contextuais, as particularidades e as subjetividades de sua intervenção estão inseridas em contextos sociais e culturais específicos. 
Apesar dos quadros conceituais propostos ainda não contemplarem as especificidades da intervenção nos esportes de aventura, verificam-se alguns componentes comuns na atividade desses profissionais, como o estabelecimento de metas para os atletas, a gestão do ambiente de treino, a avaliação da aprendizagem dos atletas e a presença de uma filosofia pessoal para fundamentar as próprias ações (COLLINS; COLLINS, 2012). Ao considerar a complexidade e o dinamismo da intervenção do treinador, independentemente da modalidade e do contexto esportivo, é possível presumir que a natureza da intervenção do TEA sugere demandas adicionais e particulares (BERRY; LOMAX; HODGSON, 2015).

As implicações da imprevisibilidade do ambiente natural de prática esportiva (a natureza) nos pensamentos do TEA, a necessidade de se manter nesse ambiente durante a intervenção, a especificidade das habilidades esportivas e o caráter individual das motivações dos atletas são demandas que tipificam a sua intervenção e que suscitam o debate conceitual (CHRISTIAN; BERRY; KEARNEY, 2017; COLLINS; COLLINS, 2012). Dessa forma, algumas iniciativas de pesquisadores da School of Sport, Tourism and The Outdoors, da University of Central Lancashire, no Reino Unido, têm buscado a definição de componentes conceituais que possam representar e direcionar o coaching nos esportes de aventura, conforme recentemente proposto pelo modelo Conceptualizing the adventure sports coach model (COLLINS; COLLINS, 2015) (Figura 1).

Figura 1 - Conceptualizing the adventure sports coach model.

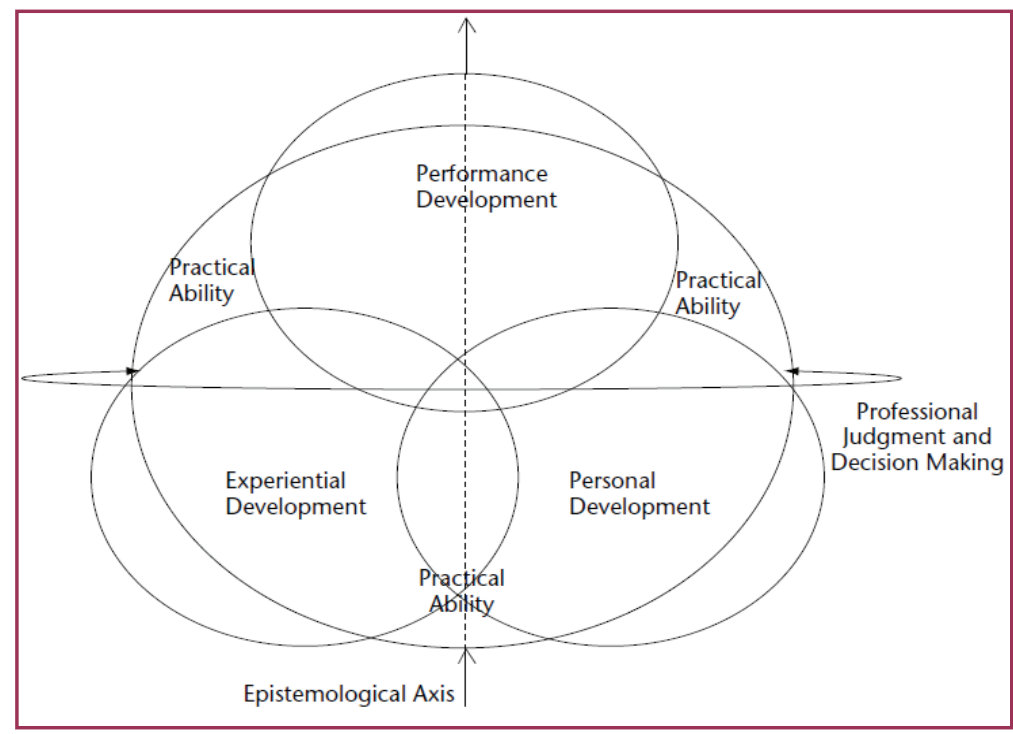

Fonte: Collins e Collins (2015, p. 22).

$\mathrm{Na}$ estruturação dessa proposta foram consideradas três prerrogativas emergentes do contexto de intervenção do TEA: a necessidade de habilidades e competências comuns entre ele e os treinadores de esportes tradicionais, e também aquelas que legitimam a sua atividade; as motivações para a prática de esportes de aventura; e os possíveis papéis assumidos pelo TEA (COLLINS; COLLINS, 2015). Essas indicações estão conceitualmente orientadas a aspectos contextuais relacionados ao foco da atividade do TEA, a processos de pensamento e à ação que fundamenta a intervenção do treinador, além da inter-relação entre esses vieses.

Por definição, os componentes do modelo compreendem: os focos da intervenção do TEA (Performance Development, Experiential Development e Personal Development) que podem também considerar domínios de resultados esperados aos praticantes; o julgamento 
profissional e a tomada de decisão (Professional Judgment e Decision Making - PJDM), que compreendem a amálgama que articula as três funções possíveis e o domínio das habilidades práticas específicas da modalidade esportiva (Practical Ability); e o eixo epistemológico (Epistemological Axis) como elemento determinante à intervenção do TEA.

\subsection{O FOCO DA INTERVENÇÃO E AS HABILIDADES ESPORTIVAS DO TEA}

A respeito do componente foco da intervenção, o TEA pode assumir, de acordo com Collins e Collins (2012), três possíveis funções com ênfases e objetivos distintos. A função de "Treinador" compreende a intervenção que enfatiza o desenvolvimento de habilidades esportivas, em que o TEA desenvolve situações de ensino/treino para favorecer o praticante a se tornar, gradativamente, mais autônomo e proficiente (Performance Development). Já na função de "Guia", o foco está na condução dos praticantes em ambientes naturais de aventura, proporcionando uma experiência pessoal de prática esportiva desejada às próprias expectativas e fins (Experiential Development). E, por último, a função de "Professor", que ocorre quando o TEA prioriza uma abordagem em que a situação de prática esportiva de aventura na natureza opera como um instrumento pedagógico para o desenvolvimento de competências, valores e atitudes do praticante, aplicáveis para além do contexto esportivo (Personal Development).

Embora possa haver sobreposições entre essas funções, cada uma delas implica abordagens de intervenção distintas, em que determinada função enfatizada pelo TEA não se dará em detrimento das demais. O que ocorre é que, geralmente, ele ocupa uma posição centralizada no modelo (ilustrado pelas áreas sobrepostas na Figura 1), deslocando-se para uma mais específica em função das motivações e expectativas do praticante, assim como das condições do ambiente natural de prática da modalidade, resultando na priorização de determinados conteúdos e procedimentos (COLLINS; COLLINS, 2012; 2015).

Apesarde as funções de guia ou professor sugerirem diferentes posturas epistemológicas (padrões de pensamento), elas exigem habilidades comuns às de treinador. Ao enfatizar qualquer uma das três funções, o TEA tem, de fato, como foco fundamental, o desenvolvimento das habilidades esportivas, o que lhe permite assumir uma abordagem que, externamente, e em circunstâncias distintas, pode aparentar estar atuando como guia, professor ou treinador. Portanto, a interface entre essas funções permite delimitar e conceituar a atividade do TEA mediante uma perspectiva mais ampla, na qual o que sustenta a oscilação entre tais funções é a prática esportiva de aventura na natureza como campo de intervenção.

Alguns estudos recentes (BRASIL et al., 2016; CHRISTIAN; BERRY; KEARNEY, 2017; COLLINS; COLLINS; GRECIC, 2015) evidenciam que os principais indicadores comuns à intervenção do TEA em distintas modalidades são a variedade de funções que ele desempenha (guia, treinador e professor), os diferentes focos da intervenção (lazer, saúde e treinamento esportivo) e também a ampla gama de motivações, faixas etárias e níveis de habilidade dos praticantes. Essas evidências contrastam com a intervenção nos esportes convencionais, nos quais o treinador está potencialmente suscetível a intervir em um contexto mais delimitado.

As nuances entre uma função e outra, enfatizadas pelo treinador, expressam, particularmente, os papéis assumidos por ele e as fronteiras entre as ações envolvidas em cada um desses papéis. Lyle (2018) sugere a noção "família de papéis" como um espaço social 
onde a intervenção do treinador está situada, esclarecendo assim o frequente equívoco de se considerar os papéis, a expertise, a certificação e a atividade profissional dentro de um espaço externamente delimitado.

No caso dos esportes de aventura, os papéis de guia, professor e treinador demandam do TEA a capacidade de manter-se no ambiente onde são desenvolvidas as atividades de coaching (mar, rio, montanha, neve, entre outros). Essa capacidade procedimental (Practical Ability) é componente da intervenção que tem relação direta com o gerenciamento do risco e com a competência pedagógica para instruir e avaliar constantemente os praticantes (COLLINS; COLLINS, 2013). O requisito é que o TEA seja autônomo no contexto de prática da modalidade, interpretando constantemente as circunstâncias do ambiente, tomando decisões adequadas e proporcionando um ambiente seguro ao praticante e a si próprio. Neste caso, o domínio das habilidades esportivas é um atributo fundamental à intervenção, em vez de corresponder apenas a um status acerca do que se é "esperado e admirado" em um treinador esportivo (COLLINS; COLLINS, 2015).

\subsection{A ESTRUTURA EPISTEMOLÓGICA DO TEA}

Além de adaptar sua intervenção à complexidade e ao dinamismo característico do coaching esportivo, o treinador dispõe de um forte quadro de referência edificado sobre seus objetivos e idealização de suas ações. Isso envolve uma organização cognitiva e uma série de decisões que permitem que ele conduza/operacionalize sua intervenção e as relações sociais entre os atores envolvidos (significados associados), momento a momento, a partir de uma base de pensamentos mais estáveis (LYLE, 2018).

Essa estrutura cognitiva, que abrange uma rede de pensamentos e decisões, tem origem nas crenças pessoais do treinador. No modelo proposto por Collins e Collins (2015), esse constructo está representado no eixo epistemológico (Epistemological Axis), também denominado, por Grecic e Collins (2013), de cadeia epistemológica (Epistemological Chain) ou crença epistemológica. Consiste em uma das dimensões da filosofia do TEA acerca de sua perspectiva de como o conhecimento é criado, construído, adquirido e desenvolvido, com importantes efeitos sobre o seu desempenho intelectual, interpretativo e compreensivo, no intuito de analisar e solucionar problemas.

Embora essa estrutura da filosofia do TEA apresente um caráter subjetivo e abrangente, ela tem implicações claras na abordagem do processo de coaching. Segundo Grecic e Collins (2013), há uma consistente relação entre a crença epistemológica do treinador, o seu modus operandi (suas ações, rotinas e as suas idealizações) e as metas que estabelece para os praticantes. De fato, o planejamento ou idealização da sessão, a criação do ambiente de aprendizagem, as instruções fornecidas e o modo de avaliar o desempenho dos praticantes são manifestações da filosofia do TEA e, de certa forma, de suas experiências e conhecimentos.

No modelo apresentado, a interação entre o eixo epistemológico (Epistemological Axis), o processo de julgamento e a tomada de decisão do TEA sustentam as diferentes funções (guia, professor e treinador) que sua intervenção perpassa (COLLINS; COLLINS; GRECIC, 2015). Quanto mais consciente de seus pensamentos, escolhas e decisões, mais facilmente o TEA será capaz de mover a abordagem de sua intervenção entre as diferentes funções, sem perder a coerência e o foco em sua meta principal. Deste modo, ele poderá orientar os praticantes 
como um guia, sobretudo em situações de risco elevado, mantendo, simultaneamente, o foco no desenvolvimento das habilidades esportivas e caracterizando sua função como treinador (BERRY; LOMAX; HODGSON, 2015).

A estrutura epistemológica do TEA, segundo Grecic e Collins (2013), pode estar posicionada em um contínuo que varia de uma estrutura mais elementar ou "ingênua" para uma mais complexa e sofisticada (Quadro 1). Um treinador com uma estrutura "ingênua" acredita que o conhecimento é simples, claro e específico; utiliza abordagens com demonstrações prescritivas e feedback excessivo; demonstra autoridade em suas relações e pensa que sua intervenção pedagógica é imutável. Já o treinador que possui uma estrutura epistemológica sofisticada acredita que o conhecimento é complexo, incerto e provisório; que pode ser construído gradualmente pelo próprio aprendiz por meio de processos de raciocínio. Neste caso, sua abordagem é mais centrada no praticante, com situações de prática esportiva espontâneas (experimentação) e perguntas de sondagem, com vistas a uma aprendizagem que dê autonomia.

Quadro 1 - Características da estrutura epistemológica do TEA.

\begin{tabular}{|c|c|}
\hline \multicolumn{2}{|c|}{ Estrutura epistemológica do TEA } \\
\hline Elementar & Sofisticada \\
\hline \multicolumn{2}{|c|}{ Epistemologia } \\
\hline $\begin{array}{l}\text { O conhecimento para intervenção é passado de } \\
\text { treinador para treinador a partir de treinadores experts. } \\
O \text { treinador é o detentor do conhecimento. }\end{array}$ & $\begin{array}{l}\text { O conhecimento para intervenção é adquirido em } \\
\text { diferentes lugares e situações (viagem constante de } \\
\text { descoberta). } \\
\text { Experimentação e reflexão são mecanismos } \\
\text { importantes para criar novos conhecimentos. } \\
\text { A perspectiva é que o conhecimento seja criado e } \\
\text { incorporado pelo praticante. }\end{array}$ \\
\hline \multicolumn{2}{|c|}{ Ambiente de aprendizagem } \\
\hline $\begin{array}{l}\text { Mestre e discípulo, regras a seguir, autocrático, } \\
\text { disciplinado, relacionamento de poder, determinado } \\
\text { pelo treinador, atleta complacente, o erro é enfatizado } \\
\text { pelo treinador. }\end{array}$ & $\begin{array}{l}\text { Ambiente de aprendizagem que favorece o atleta } \\
\text { experimentar com segurança e sem ser ridicularizado. } \\
\text { Ênfase na discussão e fluxo de idéias. }\end{array}$ \\
\hline \multicolumn{2}{|c|}{ Relações sociais } \\
\hline $\begin{array}{l}\text { Momentânea, desequilíbrio de poder, o treinador dita } \\
\text { os comportamentos desejados. }\end{array}$ & $\begin{array}{l}\text { Confiança, cuidado, duradouras, comportamentos de } \\
\text { autonomia-suportada são demonstrados pelo treinador. }\end{array}$ \\
\hline \multicolumn{2}{|c|}{ Definição de metas e objetivos } \\
\hline $\begin{array}{l}\text { São prescritas pelo treinador, subjetivas às suas próprias } \\
\text { crenças, e com dependência constante do treinador }\end{array}$ & $\begin{array}{l}\text { São definidos em discussão e negociação entre } \\
\text { treinador e atleta }\end{array}$ \\
\hline \multicolumn{2}{|c|}{ Métodos e estratégias } \\
\hline $\begin{array}{l}\text { Baseado na realização de tarefas de aprendizagem } \\
\text { pré-definidas. } O \text { atleta cumpre uma série organizada } \\
\text { de tarefas. }\end{array}$ & $\begin{array}{l}0 \text { treinador propõe episódios de aprendizagem, } 0 \\
\text { atleta com diferentes níveis de desafio e complexidade. }\end{array}$ \\
\hline \multicolumn{2}{|c|}{ Julgamentos e decisões } \\
\hline $\begin{array}{l}\text { O sucesso ou fracasso é identificado por meio de } \\
\text { marcadores tangíveis ou resultados de desempenho } \\
\text { do atleta (alterações no desempenho ténico do atleta, } \\
\text { melhoria das medidas/estatísticas realizadas pelo } \\
\text { treinador). }\end{array}$ & $\begin{array}{l}\text { Depende de como o praticante se desenvolve como } \\
\text { um atleta e como pessoa com competências para sua } \\
\text { própria vida. } \\
0 \text { treinador toma decisões considerando o seguinte } \\
\text { questionamento: "O atleta é autônomo e confiante na } \\
\text { sua capacidade para tomar suas próprias decisões?" }\end{array}$ \\
\hline \multicolumn{2}{|c|}{ Direcionamentos futuro } \\
\hline $\begin{array}{l}\text { O treinador revisa constantemente os objetivos, } \\
\text { desempenho técnico e os resultados do atleta. } \\
0 \text { treinador propõe modificações para serem } \\
\text { praticadas, reaprendidas e incorporadas pelo atleta. }\end{array}$ & $\begin{array}{l}\text { O "caminho" futuro é determinado por quão autônomo } \\
\text { e autoconfiante o atleta se sente. } \\
0 \text { treinador identifica a necessidade de orientação } \\
\text { futura ao atleta ou até a remoção de sua intervenção } \\
\text { se não for mais necessária. }\end{array}$ \\
\hline
\end{tabular}


A regularidade do uso de abordagens sofisticadas tem sido identificada em estudos com TEA de paddle sports, vela esportiva, surfe, mountain bike, escalada, alpinismo e esqui (CHRISTIAN; BERRY; KEARNEY, 2017; COLLINS; COLLINS; GRECIC, 2015), cujas evidências têm justificado a suposição de que a complexidade dos pensamentos e decisões do TEA está associada a uma identidade com esse tipo de cultura esportiva (lifestyle sports) e às circunstâncias de sua atividade como coaching. Entretanto, a seleção de indivíduos com certificação em programas ou cursos de entidades esportivas pode influenciar na homogeneidade da estrutura epistemológica desses indivíduos (GRECIC; COLLINS, 2013).

A estrutura epistemológica do TEA, portanto, apresenta implicações diretas em sua capacidade cognitiva para: reconhecer e integrar pistas sobre o ambiente; analisar circunstâncias e opções situacionais; e elaborar, mentalmente, antecipações (COLLINS; COLLINS, 2015a). De fato, isso possibilita que ele analise contextualmente (considerando o risco inerente à atividade) o potencial do praticante e o que se busca desenvolver em cada situação de prática esportiva proposta, possibilitando maior precisão às estratégias de treino (COLLINS; COLLINS, 2013; 2017).

Essas estruturas de pensamento, que abrangem um conjunto de crenças pessoais do treinador, integradas a uma série de demandas impostas pelo contexto em que ele desenvolve sua atividade profissional, compõem a sua filosofia do treinador (CASSIDY; JONES; POTRAC, 2009). Isso denota que os valores pessoais que postula à sua própria vida, assim como os valores ligados à determinada cultura esportiva, conferem características específicas ao modo como ele desenvolve seu trabalho (BARNSON, 2014). Embora esse "sistema de prioridades" se altere ao longo de sua trajetória, geralmente ele apresenta determinada regularidade em termos de valores como confiança, cooperação, compreensão e expectativa, e também, disciplina, em relação ao trabalho em equipe e comunicação, presentes no processo de coaching (CARLESS; DOUGLAS, 2011).

A estrutura conceitual proposta por Collins e Collins (2015) compreende um estágio inicial de mapeamento dos aspectos que caracterizam a intervenção profissional nos esportes de aventura. Os componentes constituintes da proposta correspondem a objetos de investigação que podem conduzir a esclarecimentos mais objetivos sobre a atividade do TEA. De fato, a análise e o debate científico sobre esses componentes têm potencial para fornecer direcionamentos à intervenção e à formação nessa área.

\section{DIRECIONAMENTOS AO DESENVOLVIMENTO PROFISSIONAL E INVESTIGAÇÃO SOBRE 0 TEA}

Alguns países, como Austrália, Nova Zelândia, Reino Unido e Portugal, já apresentam uma estrutura formal para a certificação e o desenvolvimento de profissionais que intervêm nos esportes de aventura. No Brasil, a certificação e a qualificação deles, na maioria das modalidades, estão vinculadas às suas respectivas entidades esportivas (BANDEIRA; RIBEIRO, 2015; PAIXÃO; TUCHER, 2010; VAZ et al., 2017). Embora tenha havido uma expansão global da oferta de prática esportiva e de atuação profissional no ensino e treino dos esportes de aventura, pouco se conhece sobre a intervenção e o desenvolvimento do TEA (ELLMER; RYNNE, 2018). Além disso, a consolidação desse conjunto de práticas esportivas implica a busca de níveis adequados de qualificação profissional. 
A agenda investigativa evidencia que a filosofia dos treinadores se relaciona mais com suas experiências pessoais e as características da sua intervenção do que propriamente com suas experiências formativas em contextos educacionais formais (GILBERT; TRUDEL, 2001). A análise do elo entre a estrutura epistemológica (pensamentos e idealizações) e as ações pedagógicas do treinador é um aspecto proeminente nos programas e cursos de formação na área. Entretanto, a natureza subjetiva e complexa dessa temática parece contribuir para sua ausência nesse contexto (CARLESS; DOUGLAS, 2011; CASSIDY; JONES; POTRAC, 2009).

A promoção de estratégias formativas que conduzam os próprios treinadores a apreciar como seus valores e crenças manifestam-se em seus comportamentos pode contribuir de modo efetivo na superação de um tipo de "vazio" epistemológico, no qual o conhecimento tácito é hegemônico para a eficácia do treinador (GRECIC; COLLINS, 2013). Para desenvolver a sua intervenção profissional, o treinador precisa estar ciente de seus próprios valores pessoais e crenças epistemológicas. Assim, ao confrontar, refletir, discutir e refinar suas convicções e pensamentos, pode alcançar níveis mais elevados de coaching e estar mais propenso à escolha de situações de aprendizagem profissional que estejam em conformidade com suas motivações pessoais (GILBERT; TRUDEL, 2001).

Uma compreensão detalhada sobre a epistemologia do TEA pode fornecer importantes orientações aos formadores de treinadores para identificar padrões de crenças epistemológicas a serem reestruturadas e reconhecer experiências dos próprios treinadores que sirvam de estímulo para seu engajamento deles nesse processo. Em situações formais de qualificação, explorar o que não sabe daquilo que realmente faz pode levar um treinador experiente a contextualizar novos episódios significativos de aprendizagem (CASSIDY; JONES; POTRAC, 2009).

No caso do TEA, de uma mesma modalidade ou entidade esportiva, a utilização de conceitos e vocabulário comuns possibilita compartilhar experiências e a reflexão sobre a própria intervenção pedagógica e a dos pares. A comunicação habitual entre os treinadores tem sido considerada um dos principais recursos da aprendizagem social em comunidades de prática de treinadores (CULVER; TRUDEL, 2008) e, também, no desenvolvimento de ambientes colaborativos de aprendizagem (BERTRAM; CULVER; GILBERT, 2016).

No âmbito investigativo, a realização de estudos de caráter exploratório e descritivo é recomendada, com foco em diferentes modalidades, países e realidades de intervenção, para identificar possíveis interferências contextuais na estrutura epistemológica e na abordagem de intervenção do TEA (GRECIC; COLLINS, 2013). Particularmente, os procedimentos para o gerenciamento do risco e do potencial de danos, assim como o perfil dos praticantes, são indicadores potenciais para questionar o TEA, com o objetivo de acessar as justificativas de suas prioridades e escolhas.

A iniciativa de preservar a complexidade e as características contextuais da intervenção do TEA, de acordo com Lyle (2018), aporta implicações importantes na validade e utilidade das pesquisas na área. Para isso, há a necessidade de se ter como referência a intervenção pedagógica desses profissionais, porém, sempre vislumbrando as circunstâncias nas quais ela ocorre, e não apenas em episódios isolados da atividade do treinador. 0 desafio é articular aspectos da intervenção do treinador (instrução, objetivos, decisões, entre outros) com a sua experiência e pensamentos. Neste caso, recomenda-se a adoção de metodologias etnográficas e de observação da intervenção do TEA (FRENCH, 2016). 
O estudo das características da estrutura epistemológica do TEA, a partir da análise do que o próprio treinador declara, pensa e faz, é viável, considerando as proposições de Pajares (1992), utilizadas nas investigações das crenças de professores. Nesta perspectiva, a indicação é analisar o engajamento do TEA no contexto esportivo, os valores que estão ligados à determinada prática esportiva e, também, suas razões e significados pessoais para exercer a atividade de treinador, por meio de um paradigma interpretativista, orientado por uma epistemologia subjetiva e interacionista. O propósito é obter uma descrição coerente da estrutura epistemológica dos TEA, elucidando como determinada cultura esportiva pode balizar o desenvolvimento dessas estruturas de pensamento. E ainda, de que modo as características do contexto de intervenção desses profissionais influenciam em sua abordagem de coaching.

A investigação com foco na componente estrutura epistemológica do TEA pode fornecer evidências científicas sobre os constructos fundamentais para a sua intervenção pedagógica, já consolidados na agenda investigativa sobre o professor e o treinador esportivo. Considerar o conjunto de pensamentos e decisões do TEA e suas justificativas pessoais sobre a natureza do conhecimento e da aprendizagem compreende uma importante alternativa conceitual para superar a perspectiva da intervenção profissional nos esportes de aventura fundamentada, exclusivamente, no domínio do aparato tecnológico, na instrução técnica especializada e no gerenciamento do risco. Além de auxiliar na delimitação conceitual e legitimação dessa atividade profissional, as evidências sobre essa temática podem indicar quais aspectos desse processo podem ser influenciados deliberadamente, contribuindo para a estruturação e implementação de situações formativas que atendam às demandas da intervenção nessa área (TAYLOR; MCEWAN, 2015).

\section{CONSIDERAÇÕES FINAIS}

A perspectiva conceitual apresentada para a análise e discussão, a respeito da intervenção nos esportes de aventura, integra um cenário atual de debate científico sobre a intervenção e formação profissional do treinador esportivo. A delimitação apresentada acerca dos esportes de aventura abrange as modalidades esportivas, que se distinguem dos esportes tradicionais, e que já apresentam demanda de intervenção pedagógica com ênfase na participação, iniciação e no rendimento esportivo.

O modelo conceitual sobre o Treinador de Esportes de Aventura proposto por Collins e Collins (2015) emerge de um contexto dinâmico, no qual a diversidade de conceitos e nomenclaturas tem dificultado o esclarecimento e o avanço consensual para favorecer uma legitimidade profissional aos indivíduos que atuam na área. Os componentes integrantes do modelo abrangem: os focos da intervenção do TEA indicando o caráter híbrido de sua atividade profissional; a capacidade pessoal necessária para se manter, de modo seguro, no ambiente natural de prática esportiva; o complexo processo de gestão do risco e tomada de decisão do TEA diante da imprevisibilidade do ambiente natural; e, também, uma estrutura epistemológica pessoal que exerce papel fundamental nos seus pensamentos, decisões e ações pedagógicas.

Diante das polêmicas e divergências conceituais que têm alimentado o debate científico no âmbito dos esportes de aventura e de intervenção nessa área, o itinerário conceitual apresentado neste ensaio teórico acena um potencial investigativo, sobretudo, das crenças que fundamentam intervenção pedagógica do TEA. Demarcar conceitualmente os aspectos da 
atividade profissional nessa área, preservando as particularidades do campo de intervenção, compreende uma demanda para investigações futuras, em particular, no contexto brasileiro. A possibilidade de produção de conhecimento e de diálogo entre os pesquisadores da área pode fornecer evidências acerca dessa atividade profissional com importantes implicações na qualificação e reconhecimento social dessa área de intervenção.

Embora se considere que os temas foram abordados de modo suficiente, e sem pretensão de ser uma análise exaustiva e conclusiva nas discussões efetuadas, o presente ensaio limitou-se a apresentar um percurso de conceituação e investigação, referente à intervenção nos esportes de aventura, sob determinada perspectiva conceitual que demanda verificação empírica ampla. Ademais, o itinerário proposto compreende um entendimento presente, passível de novos questionamentos e interpretações.

\section{REFERÊNCIAS}

ABRAHAM, Andy; COLLINS, Dave; MARTINDALE, Russel. The coaching schematic: validation through expert coach consensus. Journal of Sports Science, v. 24, n. 6, p. 549-564, 2006.

AURICCHIO, José Ricardo. Formação dos profissionais da cidade de Socorro - SP em atividades de aventura no âmbito do lazer. Licere, v. 20, n. 1, p. 140-160, mar. 2017.

BANDEIRA, Marília Martins; RIBEIRO, Olivia Cristina Ferreira. Sobre os profissionais da aventura: problemas da atuação na interface esporte e turismo. Licere, v. 18, n. 3, p. 116-157, set. 2015.

BARNSON, Steven. The authentic coaching model: a grounded theory of coaching. International Sport Coaching Journal, v. 1, n. 2, p. 61-74, 2014.

BERRY, Matt; LOMAX, Jane; HODGSON, Chris. Introduction. In: BERRY, Matt; LOMAX, Jane; HODGSON, Chris (Eds.). Adventure sport coaching. London: Routledge, 2015. p. 11-14.

BERTRAM, Rachael; CULVER, Diane Mary; GILBERT, Wade. Creating value in a sport coach community of practice: a collaborative inquiry. International Sport Coaching Journal, v. 3, n. 1, p. 2-16, 2016.

BETRÁN, Alberto Olivera; BETRÁN, Javier Olivera. Propuesta de una clasificación taxonómica de las actividades físicas de aventura en la naturaleza: marco conceptual y análisis de los criterios elegidos. Apunts: Educación Física y Deportes, v. 41, p. 108-123, 1995.

BOWES, Imornefe; JONES, Robyn. Working at the edge of chaos: understanding coaching as a complex, interpersonal system. The Sport Psychologist, v. 20, p. 235-245, 2006.

BRASIL, Vinicius Zeilmann et al. As ações pedagógicas para a intervenção do treinador de surf. Movimento, v. 22, n. 2, p. 403-416, abr. /jun. 2016.

BREIVIK, Gunnar. Trends in adventure sports in a post-modern society, Sport in Society, v. 13, n. 2, p. 260-273, 2010.

BRYMER, Eric. Extreme sports as a transformational tourism. In: REISINGER, Yvette (Ed.).

Transformational tourism: tourist perspectives. Wallingford: CABI, 2013. p. 111-124. 
CARLESS, David; DOUGLAS, Kitrina. Stories as personal coaching philosophy. International Journal of Sports Science and Coaching, v. 6, n. 1, p.1-12, 2011.

CARVALHINHO, Luís et al. A emergência do sector de desporto de natureza e a importância da formação. Efdeportes, Buenos Aires, v. 14, n. 140, 2010. Disponível em: <http:// www.efdeportes. com/efd140/desporto-de-natureza-e-formacao.htm>. Acesso em: 17 jan. 2018.

CASSIDY, Tania; JONES, Robyn; POTRAC, Paul. Developing a coach philosophy. In: CASSIDY, Tania; JONES, Robyn; POTRAC, Paul (Eds.). Understanding sports coaching: the social, cultural and pedagogical foundations of coaching practice. London: Routledge Abingdon, 2009. p. 55-64.

CHRISTIAN, Ed; BERRY, Matt; KEARNEY, Phil. The identity, epistemology and developmental experiences of high-level adventure sports coaches. Journal of Adventure Education and Outdoor Learning, v. 17, n. 4, p. 353-366, 2017.

COLLINS, Loel; COLLINS, Dave. Conceptualizing the adventure-sports coach. Journal of Adventure Education Outdoor Learning, v. 12, n. 1, p. 81-93, 2012.

COLLINS, Loel; COLLINS, Dave. Conceptualizing the adventure sports coach. In: BERRY, Matt; LOMAX, Jane; HODGSON, Chris (Eds.). Adventure sport coaching. London: Routledge, 2015. p. 5-23.

COLLINS, Loel; COLLINS, Dave. Decision making and risk management in adventure sports coaching. Quest, v. 65, p. 72-82, 2013.

COLLINS, Loel; COLLINS, Dave. Integration of professional judgement and decision-making in high-level adventure sports coaching practice. Journal of Sports Sciences, v. 33, n. 6, p. 622-633, 2015a.

COLLINS, Loel; COLLINS, Dave. The foci of in-action professional judgement and decisionmaking in high-level adventure sports coaching practice. Journal of Adventure Education and Outdoor Learning, v. 17, n. 2, p. 122-132, 2017.

COLLINS, Loel; COLLINS, Dave; GRECIC, David. The epistemological chain in high-level adventure sports coaches. Journal of Adventure Education and Outdoor Learning, v. 15, n.3, p. 224-239, 2015.

CÔTÉ, Jean et al. The coaching model: a grounded assessment of expert gymnastic coaches' knowledge. Journal of Sport and Exercise Psychology, v.17, p. 1-17, 1995.

CULVER, Diane; TRUDEL, Pierre. Clarifying the concept of Communities of Practice in Sport. International Journal of Sports Science and Coaching, v. 3, n. 1, p. 1-10, 2008.

CUSHION, Chris. Modelling the complexity of the coaching process. International Journal of Sports Science and Coaching, v. 2, n. 4, p. 395-401, 2007.

CUSHION, Chris; ARMOUR, Kathleen; JONES, Robyn. Locating the coaching process in practice: models 'for' and 'of' Coaching. Physical Education and Sport Pedagogy, v. 11, n. 1, p. 83-99, 2006.

DIAS, Cleber Augusto Gonçalves. Notas e definições sobre esporte, lazer e natureza. Licere, v.10, n. 3, p. 1-36, set. 2007.

DIAS, Cleber Augusto Gonçalves; MELO, Vitor Andrade de; ALVES JÚNIOR, Edmundo. Os estudos dos esportes na natureza: desafios teóricos e conceituais. Revista Portuguesa de

Ciências do Desporto, v. 7, p. 65-95, 2007. 
ELLMER, Eva; RYNNE, Steven. Professionalisation of action sports in Australia. Sport in Society, mar, 2018. Disponível em: <https://www.tandfonline.com/doi/abs/10.1080/17430437.2018. 1440700>. Acesso em: 18 mar. 2018.

FRENCH, Graham. Going pro: point of view cameras in adventure sports research. Journal of Outdoor and Environmental Education, v. 19, n. 1, p. 2-9, 2016.

FUNOLLET, Feliu. Propuesta de clasificación de las actividades deportivas en el medio natural. Apunts: Educación Física y Deportes, v.41, p.124-129, 1995.

GILBERT, Wade; TRUDEL, Pierre. Learning to coach through experience: Reflections in model youth sport coaches. Journal of Teaching Physical Education, v. 21, p. 16-34, 2001.

GRECIC, David; COLLINS, Dave. The epistemological chain: Practical applications in sports. Quest, v. 65, n. 2, p. 151-168, 2013.

INÁCIO, Humberto Luis de Deus. Práticas Corporais de Aventura na Natureza. In: GONZÁLEZ, Fernando Jaime; FENSTERSEIFER, Paulo Evaldo (Orgs.). Dicionário Crítico de Educação Física. 3. ed. ljuí: Unijuí, 2014. p. 531-535.

JONES, Robyn; WALLACE, Mike. Another bad day at the training ground: Coping with ambiguity in the coaching context. Sport, Education and Society, v. 10, n. 1, p. 119-134, 2005.

LYLE, John. Reflecting on the development of a conceptual framework for sport coaching. International Sport Coaching Journal, v.5, n.1, p. 90-98, 2018.

LYLE, John. Understanding the coaching process: a framework for social analysis. Quest, v. 54, $\mathrm{n}$. 1, p. 34-48, 2002.

MARINHO, Alcyane. Lazer, aventura e risco: reflexões sobre atividades realizadas na natureza. Movimento, v. 14, n. 2, p. 181-206, abr./jun. 2008.

MARINHO, Alcyane; UVINHA, Ricardo Ricci (Orgs.). Lazer, esporte, turismo e aventura: a natureza em foco. Campinas: Ed. Alínea, 2009.

OJALA, Anna-Liisa; THORPE, Holly. The role of the coach in action sports: using a problembased learning approach. International Sport Coaching Journal, v. 2, p. 64-71, 2015.

PAJARES, Frank. Teachers' beliefs and educational research: cleaning up a messy construct. Review of Educational Research, v. 62, n. 3, p. 307-332, 1992.

PAIXÃO, Jairo Antônio da. 0 instrutor de esporte de aventura no Brasil e os saberes necessários à sua atuação profissional. Curitiba: Ed. CRV, 2012.

PAIXÃO, Jairo Antônio da et al. Risco e aventura no esporte na percepção do instrutor.

Psicologia \& Sociedade, v. 23, n. 2, p. 415-425, 2011.

PAIXÃO, Jairo Antônio da; GEIDEL, Adriadne Runte. Percepção de instrutores com relação aos procedimentos necessários à condução de modalidades de esporte de aventura. Revista Brasileira de Ciência e Movimento, v. 26, n. 2, p. 82-91, 2018.

PAIXÃO, Jairo Antônio da; SILVA, Marcus Pires. O risco na concepção de instrutores de esporte de aventura. Psicologia \& Sociedade, v. 29, p. 1-10, 2017. 
PAIXÃO, Jairo Antônio da; TUCHER, Guilherme. Risco e aventura por entre as montanhas de minas: a formação do profissional de esporte de aventura. Revista Pensar a Prática, v. 13, n. 3 , p. 1-19, jul./set. 2010.

PIMENTEL, Giuliano Gomes de Assis. Esportes na natureza e atividades de aventura: uma terminologia aporética. Revista Brasileira de Ciências Esporte, v. 35, n. 3, p. 687-700, jul./set. 2013.

RAMOS, Valmor; BRASIL, Vinicius Zeilmann; GODA, Ciro. O conhecimento pedagógico para 0 ensino do surf. Revista de Educação Física/UEM, v. 24, n. 3, p. 381-392, out./nov. 2013

SCHWARTZ, Gisele Maria; CARNICELLI FILHO, Sandro. (Desin) Formação profissional e atividades de aventura: focalizando os guias de "Rafting". Revista Brasileira de Educação Física e Esporte, v. 20, n. 2, p. 103-109, abr./jun. 2006.

TAYLOR, Bill; MCEWAN, Islay. Professionalism and the adventure sports coach. In: BERRY, Matt; LOMAX, Jane; HODGSON, Chris (Eds.). Adventure sport coaching. London: Routledge, 2015. p. 329-349.

THORPE, Holly; WHEATON, Belinda. 'Generation X Games', Action Sports and the Olympic Movement: Understanding the Cultural Politics of Incorporation. Sociology, v. 45, n. 5, p. 830 847, 2011.

UVINHA, Ricardo Ricci Juventude, lazer e esportes radicais. Barueri: Manole, 2001.

VAZ, Jamile Machado et al. Percepção de competências profissionais de instrutores de atividades de aventura na natureza atuantes em Florianópolis/SC. Movimento, v. 23, n. 1, p. 295-310, jan./mar. 2017.

WHEATON, Belinda. Introduction. In: WHEATON, Belinda (Ed.). Understanding lifestyle sports: consumption, identity and difference. NY: Routledge, 2004. p. 1-28.

Apoio: O presente trabalho foi realizado com apoio da Coordenação de Aperfeiçoamento de Pessoal de Nível Superior - Brasil (CAPES) - Código de Financiamento 001. This study was financed in part by the Coordenação de Aperfeiçoamento de Pessoal de Nível Superior - Brasil (CAPES) - Finance Code 001. 